\title{
Éditorial
}

\section{Le jour où le cloud explosera...}

\begin{abstract}
C
ertes, le siècle commence suffisamment mal pour qu'il ne soit utile d'en rajouter une louche. Et pourtant, quitte à en achever certains, il faut la rajouter, la louche, et dénoncer une menace rampante : le numériquement correct. Une sorte de déclinaison du politiquement correct aux nouvelles technologies. Le numériquement correct, c'est ce consensus mou selon lequel toute nouveauté est forcément géniale pourvu qu'elle soit numérique. Redoutable ! Il est donc urgent d'ouvrir les yeux. Non, l'homme ne périra pas grillé par le réchauffement climatique. Il disparaîtra bien avant, réduit en bouilli, dissout en pixels, encodé, digitalisé. Oui, digéré par internet. Par cet internet qui nous ensorcelle.
\end{abstract}

Avouez que vous vous êtes tous, au moins une fois, posé la question du sens du progrès. A-t-il vraiment pris le bon chemin ? Ne se serait-il pas égaré dans une impasse, le jour où, au confluent des années 70 et 80 , il s'est engagé sur le chemin cahoteux des bits et des octets ? Ce doute, vous l'avez forcément eu la fois où, effleurant une mauvaise touche, vous avez fait disparaître tous vos fichiers de votre ordi, lequel n'a dû son salut qu'à la somme exorbitante que vous aviez dû débourser pour l'acquérir. Ce doute, vous l'avez bien sûr éprouvé lorsque votre smartphone est tombé par mégarde dans la cuvette des WC, anéantissant du même coup, photos, amis, amants, messages, musique, Candy crush, soit l'intégralité de votre vie virtuelle. Cette vie virtuelle qui, justement, a pris autant de place, sinon plus, que votre existence réelle. Songez que, bientôt, les casques de réalité virtuelle (l'oxymore le plus hallucinant qu'il nous ait été donné d'avaler), vous permettront d'avoir une double vie trépidante depuis votre canapé. Vous pourrez ainsi visiter les châteaux de la Loire en Ferrari, la main sur le genou de miss monde, puis l'emmener en hélicoptère au-dessus de la savane, lancer la baballe à un lion, faire un set avec Federer, un Scrabble avec Giscard. Après ça, comment voulez vous que le réel fasse le poids ? D’autant plus que votre vie réelle, elle, va progressivement vous échapper.

Pour ne pas avoir l'air à côté de la plaque, nous sommes tous connectés. L'étape d'après le « branché ». Une belle entourloupe ! Paradoxalement, plus vous êtes connecté, au nom de la liberté et de l'autonomie, plus vous êtes ligoté à des serveurs. Avec toutefois le soulagement de n'être pas un pauvre plouc, puisque vous apprenez en 
même temps que tout le monde que Dupont Meubles fait 50 \% sur les sofas ou que Cécile Duflot a mis une robe à fleurs. Vous êtes connecté, comme le pot de fleurs qui envoie un sms quand il n'a plus d'eau ou votre frigo, désormais capable de commander tout seul la sauce tomate au drive du coin. En contrepartie de cette dévotion à la modernité la plus cliquante, vous avez accepté d'être géolocalisé, fiché, profilé, sondé, étiqueté. Vous ne vous appartenez plus. Le big data vous fait acheter ce qu'il veut, partir en vacances là où il veut, quand il veut. Dans des autos et des avions qui savent déjà rouler ou voler tout seul. Votre taux de cholestérol, la forme de vos molaires, votre tension sont dans le cloud, tout là haut. Bientôt, des robots vous convoqueront pour changer les pièces défectueuses. Et vous croyez être encore libres de penser ? Foutaises ! Insidieusement, comme de vulgaires Coréens du nord, le politiquement correct, la bien pensance sont injectés dans votre cortex. En attendant que vous soient greffées des puces NFID entre deux neurones. Il ne vous restera bientôt plus rien d'authentique.

Sauf si, un jour, et ce jour-là est proche, les peuples se réveillent, et se mettent à suivre une sorte de Che Guevarra de la libération numérique. Ce jour-là, un éclair de lucidité parcourra la planète jusqu’à foudroyer les Google, Apple et autres Microsoft. S'élèvera alors une immense clameur de délivrance mondiale tandis que des milliards de smartphones seront jetés au fond des incinérateurs et que, dans le ciel, on entendra ce grand « Chploooufff... ! » que fait le cloud lorsqu'il implose. Passés quelques instants de désarroi, notamment chez les plus jeunes, un sentiment de légèreté s'emparera de la population, une sorte d'extase, proche, sans doute, de ce que devaient ressentir jadis les esclaves auxquels on ôtait les chaînes. Sans wifi, ni bluetooth, les gens déambuleront la tête haute, les mains dans les poches, avec l'excitation nouvelle d'être numériquement à poil. Ce jour-là, pour la première fois dans l'histoire de l’humanité, le progrès devra remballer ses trouvailles et s'avouer vaincu.

Sébastien Lacroix

Rédacteur en chef L'Union-L'Ardennais

Les opinions émises n'engagent que leurs auteurs. 